\title{
Cerebral and Extracranial Neurodegeneration are Strongly Coupled in Parkinson's Disease
}

\author{
Jörg Spiegel ${ }^{*}, 1$, Dirk Hellwig ${ }^{2}$, Wolfgang H. Jost ${ }^{3}$, Georgios Farmakis ${ }^{2}$, Samuel Samnick ${ }^{2}$, \\ Klaus Fassbender ${ }^{1}$, Carl M. Kirsch ${ }^{2}$ and Ulrich Dillmann ${ }^{1}$
}

\author{
${ }^{\prime}$ Departments of Neurology and ${ }^{2}$ Nuclear Medicine, Saarland University, D-66421 Homburg/Saar, Germany \\ ${ }^{3}$ Department of Neurology, Deutsche Klinik fur Diagnostik, D-65191 Wiesbaden, Germany
}

\begin{abstract}
In idiopathic Parkinson's disease (PD), a generalized Lewy body type-degeneration in the brain as well as extracranial organs was identified. It is unclear, whether cerebral and extracranial Lewy body type-degeneration in PD are coupled or not. To address this question, cerebral $\left[{ }^{123} \mathrm{I}\right] \mathrm{FP}-\mathrm{CIT}$ SPECT - to quantify cerebral nigrostriatal dopaminergic degeneration - and myocardial $\left[{ }^{123} \mathrm{I}\right] \mathrm{MIBG}$ scintigraphy - to quantify extracranial myocardial sympathetic degeneration were performed in 95 PD patients and 20 healthy controls. At each Hoehn and Yahr stage separately, myocardial MIBG uptake correlated significantly with striatal FP-CIT uptake. No such correlation was found in the controls. Cerebral and extracranial Lewy body type-degeneration in PD do not develop independently from each other but develop in a strongly coupled manner. Obviously cerebral and extracranial changes are driven by at least similar pathomechanisms. Our findings in controls contradict a physiological correlation between nigrostriatal dopaminergic and myocardial sympathetic function.
\end{abstract}

Keywords: Parkinson's disease, FP-CIT SPECT, MIBG scintigraphy.

\section{INTRODUCTION}

Idiopathic Parkinson's disease (PD) results from a Lewy body type-degeneration of dopaminergic neurons in the pars compacta of substantia nigra. First parkinsonian motor symptoms occur after a loss of about 60 per cent of dopaminergic nigral cells and after dopamine concentration in the posterior putamen has been reduced by about 80 per cent (Bernheimer JCS 1973; Deuschl 1999) [1, 2]. This presynaptic nigrostriatal dopaminergic deficit in $\mathrm{PD}$ can be quantified by $\left[{ }^{123} \mathrm{I}\right]$ FP-CIT SPECT. FP-CIT SPECT measures the concentration of presynaptic striatal dopamine reuptake transporters (Booij Synapse 2001) [3]. Beyond the cerebral Lewy body type-degeneration, numerous studies found a Lewy body type-degeneration outside the brain in $\mathrm{PD}$, too, primarily in the extracranial autonomous nervous system (Edwards Neurology 1992, Iwanaga Neurology 1999, Kaufmann Neurology 2004, Micieli NS 2003, Okada PI 2004, Wakabayashi EJ 1997) [4-9]. These studies show that PD does not affect only the brain. It is unclear, whether cerebral and extracranial Lewy body type-degeneration in PD are coupled or not.

$\left[{ }^{123}\right.$ I]MIBG scintigraphy measures the function of extracranial myocardial postganglionic sympathetic innervation. $\left[{ }^{123} \mathrm{I}\right] \mathrm{MIBG}$ is a radioiodinated analogue of noradrenaline (norepinephrine). It is actively transported into noradrenaline granules of sympathetic nerve terminals by the noradrenaline transporter (Wieland JNM 1980) [10]. [223]]MIBG scintigraphy reveals a high sensitivity concerning PD because it discloses an impaired myocardial postganglionic sympathetic innervation in PD (Braune CAR 2001, Hamada JNNP 2003, Yoshita NMC 1998) [11-13]. These data suggest that MIBG

*Address correspondence to this author at the Department of Neurology Saarland University, D-66421 Homburg/Saar, Germany;

E-mail: joerg.spiegel@uniklinik-saarland.de scintigraphy allows estimating the extent of Lewy body typedegeneration of the cardiac plexus, exemplarily of an extracranial structure.

Since there exists no information about the correlation between cerebral and extracranial Lewy body typedegeneration in PD, we applied FP-CIT SPECT and MIBG scintigraphy to address this point in PD. We used FP-CIT SPECT/MIBG scintigraphy to quantify nigrostriatal dopaminergic/myocardial sympathetic degeneration. We looked for a correlation between cerebral nigrostriatal degeneration and extracranial myocardial sympathetic degeneration. Such a correlation would indicate that 1) cerebral nigrostriatal dopaminergic and extracranial myocardial sympathetic Lewy body type-degeneration are coupled and that 2) both degenerative processes result from identical or at least similar processes. Since stage, severity and duration of PD correlate significantly with reduction of nigrostriatal FP-CIT uptake (Benamer MD 2000) [14] and myocardial MIBG uptake (Hamada JNNP 2003) [12], we evaluated PD patients separately after Hoehn and Yahr stage to exclude a statistical bias due to the factors disease stage or severity.

\section{PATIENTS AND METHODS}

\section{Patients and controls}

\section{Patients}

The study involved 95 patients with idiopathic Parkinson's disease (PD; age $59 \pm 11$ years, mean $\pm \mathrm{SD}$, further details in Table 1). PD was diagnosed according to the criteria of the UK Parkinson's Disease Society Brain Bank (Hughes JNNP 1992) [15]. Two different neurologists examined the patients. No patient was found to have a history of neuroleptic intake or other identifiable possible causes for secondary Parkinsonism. Cerebral MRI findings were normal in all patients, particularly regarding the absence of ei- 
ther white matter lesions or ventricular widening. Therapy with selegiline was discontinued at least 18 hours before FPCIT application due to possible interaction of its metabolites with striatal FP-CIT binding (Laruelle Synapse 1993) [16]. Further antiparkinsonian medication was continued during the time of scintigraphic examinations. Aware of the problem facing FP-CIT SPECT and MIBG scintigraphy in patients treated with antiparkinsonian drugs, the present data (Ahlskog MD 1999, Guttman Neurology 2001, Innis MD 1999, Laruelle Synapse 1993, Nurmi JCBFM 2000, Satoh JCN 1999, The Parkinson Study Group JAMA 2002) [16-22] did not allow a clear conclusion concerning the effect of dopaminergic drugs, neither on dopamine transporter binding, nor on MIBG distribution.

Table 1. Demographic Data of Patients

\begin{tabular}{|l|c|c|c|}
\hline & H\&Y 1 & H\&Y 2 & H\&Y 3+4 \\
\hline \hline $\mathrm{N}$ & 63 & 12 & 20 \\
\hline Females & 27 & 3 & 11 \\
\hline Males & 36 & 9 & 9 \\
\hline Age (range) & $37-78$ & $40-71$ & $42-76$ \\
\hline Age & $58 \pm 10$ & $56 \pm 11$ & $63 \pm 8$ \\
\hline UPDRS part III & $11 \pm 2$ & $18 \pm 3$ & $30 \pm 9$ \\
\hline
\end{tabular}

H\&Y = Hoehn and Yahr stage (Hoehn Neurology 1967) [27]. No patient was at H\&Y stage 5. Due to the case number of patients at H\&Y stage $4(n=5)$ we combined the patients at H\&Y stage $3(n=15)$ and $H \& Y$ stage $4(n=5)$ to one single group. $N=$ number of patients. UPDRS part III = part III (motor part) of the Unified Parkinson's disease rating scale (UPDRS, Fahn 1987) [28]. Antiparkinsonian medication had been stopped 12 hours before UPDRS scale was rated. Unless otherwise declared, all values are represented as mean $\pm \mathrm{SD}$.

20 subjects ( 9 females, 11 males, age $37-74$ years, $57 \pm$ 11 years) underwent FP-CIT SPECT and MIBG scintigraphy because they were referred by the outpatient physician, who assumed a beginning PD. After PD had been excluded by clinical criteria and by the further clinical course, these subjects served as controls for the correlation between FP-CIT SPECT and MIBG scintigraphy. 11 of these 20 subjects were suffering from essential tremor, 2 from psychogenic Parkinsonism, and 7 had no neurological or psychiatric disease. These controls were not significantly different from PD patients concerning age $(\mathrm{p}=0.93$; unpaired t-test). The norm values for FP-CIT SPECT and MIBG scintigraphy were not obtained from these controls but from other controls (see below).

In regard to the scintigraphic examinations, patients or controls with a clinical history of angina pectoris, arrhythmia, myocardial infarct, hypothyroidism, diabetes mellitus, cerebrovascular events, psychiatric disorders (except psychogenic Parkinsonism), malignancy, hypertension, phaeochromocytoma or any other disease, that might affect uptake of myocardial $\left[{ }^{123} \mathrm{I}\right] \mathrm{MIBG}$, were excluded. No subject was treated by tricyclic antidepressant drugs or other serotonin reuptake inhibitors, reserpine, clonidine, amphetamines, phenylpropanolamine, amiodarone or substances inducing any blockade of calcium channels. Written informed consent was obtained from all subjects prior to examination. The protocol was approved by the local ethics committee (Ethikkommission der Ärztekammer des Saarlandes).

\section{Data Acquisition $I^{123}$ IJFP-CIT SPECT}

Cerebral SPECT imaging was performed with iodine 123 ioflupane $\left(\mathrm{DaTscan}^{\circledR}\right.$, Amersham Cygne, Eindhoven, The Netherlands). 4 hours following thyroid gland blocking and intravenous injection of the radioactive substance (110 - 185 MBq, specific activity: $580-1040 \mathrm{GBq} / \mathrm{mg}$ ) cerebral SPECT images were obtained. A triple-head gamma camera (Siemens Multispect, MS 3) equipped with low-energy highresolution collimators was used. Data was acquired in a 128 x 128 matrix covering 120 degrees per camera and head, 50 seconds per view. A total of 120 views was acquired. Energy discrimination was centered in $158 \mathrm{keV}$ with a $15 \%$ window. Image filtering (Butterworth, Cutoff 0.5) was followed by attenuation correction (Chang, $0.11 / \mathrm{cm}$ ). Registration and semiquantitative analysis were performed with a workstation (HERMES, Nuclear Diagnostics, Stockholm, Sweden) and a software named BRASS (Nuclear Diagnostics, Stockholm, Sweden), modified for this purpose. The caudate nucleus, putamen and occipital lobe binding of $\left[{ }^{123} \mathrm{I}\right] \mathrm{FP}-\mathrm{CIT}$ was assessed semiquantitatively by a regions of interest (ROI) technique and compared with a control group. The ROI's included 524 pixels (according volume $=4.2 \mathrm{~cm}^{3}$ ) for each caudate nucleus, 689 pixels (according volume $=5.5 \mathrm{~cm}^{3}$ ) for each putamen, and 9008 pixels (according volume $=72$ $\mathrm{cm}^{3}$ ) for both occipital cortices together. We regarded a small central area within each nucleus and did not consider the whole nucleus to avoid partial volume and resolution effects. We calculated the ratio caudate nucleus/occipital lobe binding and the ratio putamen/occipital lobe binding. Our norm values obtained from controls were previously described (Spiegel JNNT 2007) [23].

\section{$I^{123}$ IJMIBG Scintigraphy}

In a time period of 4 to 7 days preceding or following FP-CIT SPECT of the brain, MIBG examination of the heart took place: Following thyroid gland blocking with sodium, perchlorate intravenous injection of $\left[{ }^{123} \mathrm{I}\right] \mathrm{MIBG}$ (Amersham, Amersham Buchler, Braunschweig, Germany) with a specific activity of $259-370 \mathrm{MBq} / \mathrm{mg}$ was given. The injected doses of MIBG ranged from 180 to $250 \mathrm{MBq}$. Planar wholebody images in anterior and posterior views were obtained 4 $\mathrm{h}$ after the injection using a dual-head large-field camera (Siemens Multispect, MS 2) equipped with low-energy highresolution parallel-hole collimators. MIBG uptake of myocardium and mediastinum was quantified after drawing of a rectangular region of interest over the upper mediastinum and an irregularly configured region outlining the whole heart. Then the heart-to-mediastinum (H/M) count-ratio was calculated, which was defined as the average cts/pixel in the myocardium divided by that of the upper mediastinum. Our norm values obtained from controls were previously described (Spiegel MD 2005) [24]. Within one day after MIBG imaging, additional myocardial rest perfusion imaging was performed afterwards with ${ }^{99 \mathrm{~m}}$ Tc-Sestamibi (MIBI, Cardiolite $^{\circledR}$, Bristol Myers Squibh, USA, $500 \mathrm{MBq}$ ). This perfusion imaging was used to exclude a myocardial hypoperfusion that may influence MIBG imaging adversely. Patients with an impaired myocardial perfusion were excluded from further investigation. 


\section{Statistical Analysis}

Correlations were calculated using the Pearson's correlation coefficient in case of a normally distributed data and Spearman's correlation coefficient in case of a not normally distributed data.

\section{RESULTS}

\section{Patients}

At all Hoehn and Yahr stages, caudate nucleus (CN) and putamen (PUT) FP-CIT uptake contralateral to the clinically more affected body side ( = contralateral uptake) were significantly smaller than CN/PUT FP-CIT uptake ipsilateral to the clinically more affected body side ( = ipsilateral uptake). Contralateral $\mathrm{CN}$ and/or PUT uptake was pathologically reduced in all patients.

MIBG scintigraphy disclosed a pathologically reduced myocardial MIBG uptake in 88 of 95 patients. All patients with normal MIBG scintigraphy were at Hoehn and Yahr stage 1. At each Hoehn and Yahr stage separately, myocardial MIBG uptake correlated significantly with contralateral $\mathrm{CN}$ and PUT FP-CIT uptake ( $<<0.05$; Fig. 1). Furthermore, there was a significant correlation between MIBG uptake and ipsilateral CN and PUT FP-CIT uptake ( $\mathrm{p}<0.05$; Pearson's correlation) at each Hoehn and Yahr stage. Multiple regression analysis showed that the correlation CN or PUT FP-CIT uptake versus myocardial MIBG uptake were not significantly influenced by the factors age, age at disease onset, disease duration or UPDRS ( $\mathrm{p}>0.05$; multiple regression analysis).

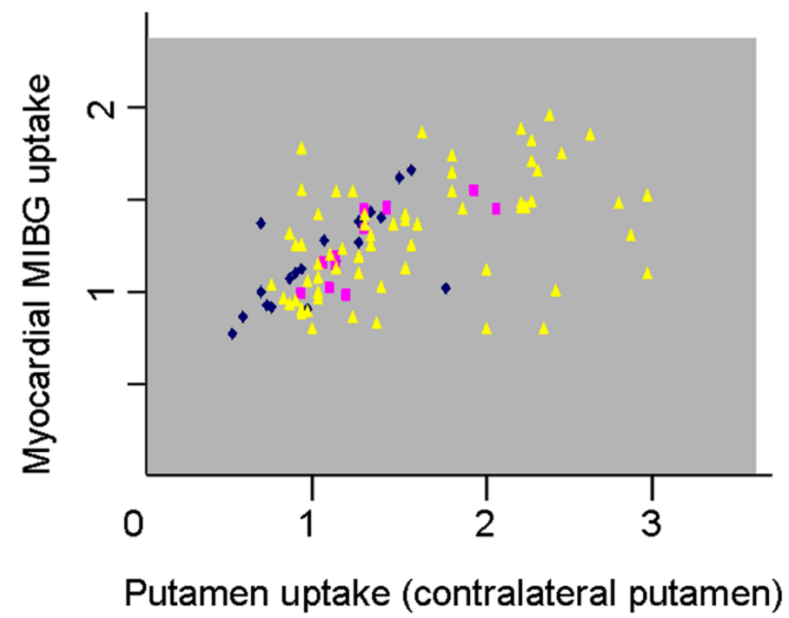

Fig. (1). Correlation between putamen FP-CIT SPECT and MIBG scintigraphy. Each patient is represented by one symbol (patients with Hoehn and Yahr stage 1: yellow triangles, patients H\&Y stage 2: red squares, patients $H \& Y$ stages $3+4$ : blue rhombs).

\section{Controls}

FP-CIT SPECT ( $\mathrm{n}=40$ values due to bilateral measurement) and MIBG scintigraphy $(n=20)$ were normal in all 20 controls. For calculation of correlation between myocardial MIBG uptake and striatal FP-CIT uptake we considered the $\mathrm{CN}$ and PUT tracer uptake each of both sides: there was no significant correlation between MIBG uptake versus $\mathrm{CN}$ or PUT FP-CIT uptake.

\section{DISCUSSION}

Idiopathic Parkinson's disease (PD) is defined by the neuropathological finding of Lewy bodies (LB) primarily in the substantia nigra and other nuclear grays of the brain (Turner 1968) [25]. Based on the intracerebral formation of LB, Braak et al. (Braak CTR 2004) [26] defined six stages of PD. This sequence of cerebral LB degeneration is not random but results from the differing vulnerability and susceptibility of cerebral cell types to develop LB (Braak CTR 2004) [26]. Numerous studies found LB also in extracranial organs, especially in the peripheral autonomic nervous system: in the sympathetic system (intermediolateral nucleus of the thoracic cord and paravertebral sympathetic ganglia), the parasympathetic system (dorsal vagal and sacral parasympathetic nuclei; Iwanaga Neurology 1999, Kaufmann Neurology 2004, Wakabayashi and Takahashi EN 1997) [5, 6, 9], the adrenal medulla, and in the neural plexi innervating the gut, heart and pelvis (Edwards Neurology 1992, Micieli NS 2003, Okada PI 2004) [4, 7, 8]. These numerous histopathological changes at different parts of central and peripheral nervous system correlate with common clinical symptoms in PD such as constipation, orthostatic hypotension, detrusor hyperactivity or seborrhea (Deuschl 1999; Micieli NS 2003) $[2,7]$. It is quite unclear whether there exists any relation between cranial and extracranial LB degeneration in PD. Furthermore the question arises whether cranial and extracranial LB degeneration are induced by the same pathomechanisms and thus are coupled in PD.

In the present paper we studied whether there exists a correlation between the cerebral nigrostriatal dopaminergic function and the extracranial sympathetic myocardial function. We quantified cerebral nigrostriatal dopaminergic function with FP-CIT SPECT and extracranial sympathetic myocardial function with MIBG scintigraphy. We assumed that nigrostriatal dopaminergic function reflects the extent of nigral LB degeneration. Accordingly we measured the sympathetic myocardial function as a correlative for severity of myocardial sympathetic LB degeneration. Since stage, severity and duration of PD correlate significantly with reduction of nigrostriatal FP-CIT uptake (Benamer MD 2000) [14] and myocardial MIBG uptake (Hamada JNNP 2003) [12], we studied patients separately after Hoehn and Yahr stage to exclude a statistical bias due to the factors disease stage or severity.

We found a highly significant correlation between cerebral caudate nucleus (CN) and putamen (PUT) FP-CIT uptake versus extracranial myocardial MIBG uptake at all Hoehn and Yahr stages. Multiple regression analysis showed this significant correlation was not caused or influenced by the factors age, age at disease onset, disease duration or UPDRS. In the non-parkinsonian control subjects, there was no significant correlation between CN/PUT FP-CIT uptake versus extracranial myocardial MIBG uptake. This contradicts a physiological coupling of nigrostriatal dopaminergic and myocardial sympathetic function and proves that the close coupling between nigrostriatal degeneration and myocardial sympathetic degeneration in PD represents a pathological phenomenon. 


\section{CONCLUSIONS}

Our data indicate a strongly coupled degeneration of nigrostriatal dopaminergic and myocardial sympathetic transmission at early as well as advanced stages of PD. We assume that at least cerebral nigrostriatal dopaminergic LB degeneration and extracranial myocardial sympathetic LB degeneration develop in a coupled manner. Obviously both processes are driven by identical or at least similar pathomechanisms. Our results give strong evidence that the clinically and histopathologically manifest cerebral and extracranial degenerative changes in PD do not develop independently from each other but develop in a strongly coupled manner.

\section{REFERENCES}

[1] Bernheimer H, Birkmayer W, Hornykiewicz O, Jellinger K, Seitelberger F. Brain dopamine and the syndromes of Parkinson and Huntington. J Neurol Sci 1973; 20(4): 415-55.

[2] Deuschl G, Krack P. Morbus Parkinson. In: Hopf HC, Deuschl G, Diener HC, Reichmann H, eds. Neurologie in Praxis und Klinik. Georg Thieme Verlag, Stuttgart New York (3rd edition); 1999 Vol 2: 49-69.

[3] Booij J, Bergmans P, Winogrodzka A, Speelman JD, Wolters EC. Imaging of dopamine transporters with [123I]FP-CIT does not suggest a significant effect on age on the symptomatic threshold of disease in Parkinson's disease. Synapse 2001; 39(2): 101-8.

[4] Edwards LL, Quigley EM, Pfeiffer RF. Gastrointestinal dysfunction in Parkinson's disease: frequency and pathophysiology. Neurology 1992; 42(4): 726-32.

[5] Iwanaga K, Wakabayashi K, Yoshimoto M, et al. Lewy body-type degeneration in cardiac plexus in Parkinson's and incidental Lewy body diseases. Neurology 1999; 52(6): 1269-71.

[6] Kaufmann H, Nahm K, Purohit D, Wolfe D. Autonomic failure as the initial presentation of Parkinson's disease and dementia with Lewy bodies. Neurology 2004; 63(6): 1093-5.

[7] Micieli G, Tosi P, Marcheselli S, Cavallini A. Autonomic dysfunction in Parkinson's disease. Neurol Sci 2003; 24(suppl 1): S32-4.

[8] Okada Y, Ito Y, Aida J, Yasuhara M, Ohkawa SI, Hirokawa K. Lewy bodies in the sinoatrial nodal ganglion: clinicopathological studies. Pathol Intern 2004; 54(9): 682-7.

[9] Wakabayashi K, Takahashi H. Neuropathology of autonomous nervous system in Parkinson's disease. Eur Neurol 1997; 38(suppl 2): $2-7$.

[10] Wieland DM, Wu J, Brown LE, Mangner TJ, Swanson DP, Beierwaltes WH. Radiolabeled adrenergic neuron-blocking agents: adrenomedullary imaging with [123I]iodobenzylguanidine. J Nucl Med 1980; 21(4): 349-53.

[11] Braune S. The role of cardiac metaiodobenzylguanidine in the differential diagnosis of parkinsonian syndromes. Clin Auton Res 2001; 11(6): 351-5.

[12] Hamada K, Hirayama M, Watanabe H, et al. Onset age and severity of motor impairment are associated with reduction of myocardial (123I)-MIBG uptake in Parkinson's disease. J Neurol Neurosurg Psychiatry 2003; 74(4): 423-6.
[13] Yoshita M, Hayashi M, Hirai S. Decreased myocardial accumulation of I123-meta-iodobenzylguanidine in Parkinson's disease. Nucl Med Commun 1998; 19(2): 137-42.

[14] Benamer HTS, Patterson J, Wyper DJ, Hadley DM, MacPhee GJA, Grosset DG. Correlation of Parkinson's disease severity and duration with 123I-FP-CIT SPECT striatal uptake. Mov Disord 2000; 15(4): 692-8.

[15] Hughes A, Daniel SE, Kilford L, Lees AJ. Accuracy of clinical diagnosis of idiopathic Parkinson's disease: a clinico-pathological study of 100 cases. J Neurol Neurosurg Psychiatry 1992; 55(3): $181-4$.

[16] Laruelle M, Baldwin RM, Malison RT, et al. SPECT imaging of dopamine and serotonin transporters with [123I]B-CIT: pharmacological characterization of brain uptake in non-human primates. Synapse 1993; 13(4): 295-309.

[17] Ahlskog JE, Uitti RJ, O'Connor MK, et al. The effect of dopamine agonist therapy on dopamine transporter imaging in Parkinson's disease. Mov Disord 1999; 14(6): 940-6.

[18] Guttman M, Stewart D, Hussey D, Wilson A, Houle S, Kish S. Influence of L-dopa and pramipexole on striatal dopamine transporter in early PD. Neurology 2001; 56(11): 1559-64.

[19] Innis RB, Marek KL, Sheff K, et al. Effect of treatment with Ldopa/carbidopa or L-selegiline on striatal dopamine transporter SPECT imaging with [123I]B-CIT. Mov Disord 1999; 14(3): 43642.

[20] Nurmi E, Bergman J, Eskola O, et al. Reproducibility and effect of levodopa on dopamine transporter function measurements: a [18F]CFT PET study. J Cereb Blood Flow Metab 2000; 20(11): 1604-9.

[21] Satoh A, Serita T, Seto M, et al. Loss of 123I-MIBG uptake by the heart in Parkinson's disease: Assessment of cardiac sympathetic denervation and diagnostic value. J Nucl Med 1999; 40(3): 371-5.

[22] The Parkinson Study Group. Dopamine transporter brain imaging to assess the effects of pramipexole $v s$ levodopa on Parkinson's disease progression. JAMA 2002; 287(13): 1653-61.

[23] Spiegel J, Hellwig D, Samnick S, et al. Striatal FP-CIT uptake differs in the subtypes of early Parkinson's disease. J Neural Transm 2007; 114(3): 331-5.

[24] Spiegel J, Möllers MO, Jost WH, et al. FP-CIT and MIBG scintigraphy in early Parkinson's disease. Mov Disord 2005; 20(5): 55261.

[25] Turner B. Pathology of paralysis agitans. In: Vinken PJ, Bruyn GW, eds. Handbook of Clinical Neurology. North-Holland Publishing Company, Amsterdam; John Wiley\&Sons Inc. New York; 1968 Vol 6: 212-7.

[26] Braak H, Ghebremedhin E, Rüb U, Bratzke HJ, Del Tredici K. Stages in the development of Parkinson's disease-related pathology. Cell Tissue Res 2004; 318(1): 121-34.

[27] Hoehn MM, Yahr MD. Parkinsonism: onset, progression and mortality. Neurology 1967; 17(5): 427-42.

[28] Fahn S, Elton RL and members of the UPDRS Development Committee. Unified Parkinson's disease rating scale. In: Fahn S, Marsden CD, Calne DB, Goldstein M, eds. Recent developments in Parkinson's disease. Macmillan Healthcare Information, Florham Park, HJ; 1987: 153-64. 